\title{
Tiempo, conocimiento y heterotopía: estéticas del jardín en la novela de J. W. Goethe Die Wahlverwandtschaften
}

\author{
Javier SÁNCHEZ-ARJONA VOSER \\ Universidad Complutense de Madrid \\ jsanchezarjona@gmail.com
}

Recibido: 10 de diciembre de 2010

Aceptado: 9 de febrero de 2011

\section{RESUMEN}

La novela de Johann Wolfgang Goethe Die Wahlverwandtschaften, publicada en 1809, es también una manera de articular conocimiento en torno a un hecho anecdótico, de forma que alcance un carácter simbólico. El centro en torno al que pivotan anécdota y conocimiento -o sea, la trama de la novela- es el jardín que se extiende en torno al castillo del barón Eduard.

Este conocimiento se archiva, se colecciona, se proyecta, se reorienta y se realiza en la novela. Todas estas acciones, cuyo objeto directo común es el jardín entendido como conocimiento, implican un modo de articular y presentar el tiempo. Por ese motivo será éste el asunto central del presente artículo. Asunto que abordaremos desde dos perspectivas: una, filosófica y otra, poetológica. Todo ello nos llevará a concluir que en el modo etimológicamente estético de entender el jardín en la novela se combinan elementos sustanciales que lo convierten en una heterotopía.

Palabras clave: Goethe, Wahlverwandtschaften, jardín, poetología del conocimiento, heterotopía.

\section{Time, Knowledge and Heterotopia: Garden-aesthetics in J.W. Goethe's Novel Die Wahlverwandtschaften}

\begin{abstract}
Goethe's novel Die Wahlverwandtschaften, published in 1809, is also a way of articulating knowledge around an anecdotal event, so that it may reach a symbolic nature. The centre around which anecdote and knowledge turn - that is the plot of the novel - is the garden around the castle of the baron Eduard.

This knowledge is filed, collected, planed, reorientated and put into practice in the novel. All these actions, whose common direct object is the garden - understood as knowledge -, involve a way of formulating and presenting time. That is the reason why I conceive the relation between time and garden as the main matter of this article. A subject I will discuss regarding it from two different points of view: the first, philosophical, the second, from the field of Poetics. All of this will lead to the conclusion that the aesthetic - in an etymological sense - way of understanding the garden in the novel results form the combination of some crucial elements that turn it into a heterotopia.
\end{abstract}

Keywords: Goethe, Wahlverwandtschaften, Garden, Poetics of Knowledge, Heterotopia.

SUMARIO: 1. Introducción. 2. Injerto y ventana: estéticas económicas de lo industrioso y de lo contemplativo. 3. Tiempo y naturaleza: paseos por el Faust y por Die Wahlverwandtschaften. 4. Tiempo, naturaleza, historia, diégesis. 5. Coleccionistas: tiempo, acumulación y la estética de la obra de arte viva. 6. Conclusión: heterotopía y estética del jardín. 
"Doch an Blumen fehlt's im Revier Sie nimmt geputzte Menschen dafür" J. W. Goethe, Faust I

"Dich verwirret, Geliebte, die tausendfältige Mischung dieses Blumengewühls über den Garten umher" J. W. Goethe, Die Metamorphose der Pflanzen

\section{Introducción: jardín y poetología del saber}

En su obra sobre Michel Foucault, Gilles Deleuze subrayó la importancia de los aportes del pensador francés con las siguientes palabras:

L'essentiel n'est pas d'avoir surmonté une dualité science-poésie que grevait encore l'œuvre de Bachelard. Ce n'est pas non plus d'avoir trouvé un moyen de traiter scientifiquement des textes littéraires. C'est d'avoir découvert et arpenté cette terre inconnue où une forme littéraire, une proposition scientifique, une phrase quotidienne, un non-sens schizophrénique, etc., sont également des énoncés pourtant sans commune mesure, sans aucune réduction ni équivalence discursive. Et c'est ce point qui n'avait jamais été atteint, par les logiciens, les formalistes ou les interprètes. Science et poésie sont également savoir (Deleuze 1986: 28-29).

La novela de Johann Wolfgang Goethe Die Wahlverwandtschaften, publicada en 1809, es también -y, por supuesto, no sólo- una forma de conocimiento. O para ser más exactos: una manera de articular conocimiento en torno a un hecho anecdótico ${ }^{1}$ de forma que alcance un carácter simbólico y llegue a ser un "caso eminente" siguiendo la argumentación de Goethe en su conocido Symbolbrief, dirigido a Schiller en 1797. El centro en torno al que pivotan anécdota y conocimiento -o sea, la trama de la novela- son los terrenos que se extienden en torno al castillo del barón Eduard y especialmente su jardín.

Este es el punto de partida desde el que pretendemos analizar la presencia del jardín ${ }^{2}$ en Die Wahlverwandtschaften, profundizando en la línea apuntada por Vogl en su obra Kalkül und Leidenschaft. Poetik des ökonomischen Menschen (2008) y añadiendo así un fragmento al país del abanico de enfoques desde los que se ha analizado este asunto en la literatura crítica ${ }^{3}$.

${ }^{1}$ No olvidemos que la novela fue concebida en un primer momento como Novelle dentro del ciclo de los Wanderjahre (BoLZ 1997: 153).

${ }^{2}$ Nótese, ya de entrada, que el jardín es un elemento recurrente en la obra de Goethe. Pensemos, por ejemplo, en Die Leiden des jungen Werthers, en Torquato Tasso, en Die natürliche Tochter o en Die Aufgeregten.

${ }^{3}$ Trazando una brevísima panorámica de la literatura crítica sobre el tema -dado que un listado exhaustivo sobrepasaría los límites del presente artículo-, vemos que el espectro de los diferentes enfoques desde los que se ha analizado la presencia del parque en Die Wahlverwandtschaften, discurre 
En la introducción a su estudio Vogl esboza las líneas maestras de un enfoque metodológico que etiqueta, siguiendo la estela de Foucault, como poetología del conocimiento. Este tipo de acercamiento parte de las siguientes premisas sobre la literatura, que se entiende según Vogl (2008: 15) como:

- Forma específica de conocimiento.

- Objeto de conocimiento.

- Elemento funcional del conocimiento.

- Producto de una organización del conocimiento.

Partimos de la base de que esta cuádruple perspectiva es perfectamente aplicable al elemento del jardín en la novela Die Wahlverwandtschaften. El jardín es una forma específica de conocimiento dado que, tanto por el modo en que se reforma como por la forma en que se posicionan los personajes frente a él, nos da información sobre el contexto en el que se desarrolla el espacio textual y sobre los protagonistas y sus relaciones interpersonales. El jardín es objeto de conocimiento, en tanto que se estudia y se convierte así en objeto de experimentación. Es elemento funcional del conocimiento, en tanto que permite demostrar en él los resultados de enfoques científicos o estéticos. $\mathrm{Y}$ es producto de una organización del conocimiento en la medida en que los cambios que se hicieron en el pasado o que se vienen haciendo a lo largo de la novela se van archivando y recordando de diferentes maneras, al tiempo que van modelando y transformando el jardín. En definitiva, el jardín es, en Die Wahlverwandtschaften, poesía -en tanto que creación- y conocimiento -en tanto que producto estético, esto es, producto de percepción-.

Este conocimiento se archiva, se colecciona, se proyecta, se reorienta y se realiza en la novela. Todas estas acciones, cuyo objeto directo común es el jardín entendido como conocimiento, implican un modo de articular y presentar el tiempo. Por ese motivo será éste el asunto central del presente artículo: la relación entre el jardín y el tiempo. Asunto que abordaremos desde dos perspectivas: una, filosófica apoyándonos sobre todo en Matussek (1998)-y otra, poetológica -siguiendo a Vogl (2008)-. Todo ello, incluyendo una breve reflexión sobre la importancia poetológica

desde el más sociológico ensayado por GERNDT (1991) hasta el estético de POLIANSKI (2004). A pesar de lo ingente de este literatura crítica, sí que cabe resumir que la mayor parte de los estudios tratan este asunto desde una perspectiva histórica, poniendo sobre todo el acento en la relación entre la presencia del parque en la novela con el fenómeno de la Gartenrevolution o con las relaciones sociales y afectivas entre sus protagonistas. Ahora bien: en los últimos años se han concentrado los esfuerzos en localizar teórica y sistemáticamente el modo en que inserta la trama en torno al parque en la novela dentro de la obra de Goethe y en su relación de trabajo sobre todo con Schiller. Merecen ser nombrados el modo en que SAMPAOLO (2003) aborda la relación entre mito y crítica a la modernidad que le era contemporánea a Goethe; el trabajo de POLIANSKI (2004), centrado en la implicación de Goethe en la creación del jardín botánico de Jena; el estudio de la presencia de la labor desarrollada por Goethe en el Ilmpark llevado a cabo por SCHNEIDER (2004); o las páginas que le dedica a Die Wahlverwandtschaften TABARASI (2007) en las que relaciona la novela con la simbología de la Gartenrevolution. En esta línea creemos que abunda la contribución que hacemos en este artículo. 
del coleccionismo en la novela, nos llevará a concluir que en el modo etimológicamente estético de entender en la novela el jardín como conocimiento se combinan elementos sustanciales de las heterotopías, según las definió Foucault en su conferencia Des espaces autres (Foucault 1994).

\title{
2. Injerto y ventana: estéticas económicas de lo industrioso y de lo contemplativo
}

\author{
"Tout se résume dans l'Esthétique et l'Économie politique" \\ S. Mallarmé, La musique et les lettres
}

La novela Die Wahlverwandtschaften empieza con un injerto: una tarde de primavera el barón Eduard acaba de concluir una productiva operación de empalme en troncos jóvenes de unos brotes recién adquiridos. Extasiado, contempla su obra acabada. En ese momento entra el jardinero y comparte la contemplación con su señor. Éste pregunta a su criado dónde se encuentra su señora. "Drüben in den neuen Anlagen" (Goethe 2009: 7) ${ }^{4}$. Así de sutilmente se plantea una dicotomía que vertebrará a lo largo de todo el texto la polaridad entre lo viejo y lo nuevo, entre lo acabado -y cerrado- y lo abierto -inconcluso, por recién comenzado-. Esta polaridad será la que definirá los dos espacios en los que quedará dividido el jardín en torno al castillo del barón Eduard desde el momento en que Charlotte, su (nueva) mujer al comienzo de la novela, empieza a desarrollar un jardín inglés más allá del huerto que había heredado y continuado Eduard. Una polaridad que, como lo han mostrado Gerndt (1991) y Niedermeier (1993), está influida por el movimiento alternativo de la sístole y la diástole empleado por Goethe en su teoría morfológica. Como lo resume Tabarasi: "Der formale Garten ist ein Produkt der Systole (des Sich-Zusammenziehens), der Landschaftsgarten einer der Diastole (des SichÖffnens)" (Tabarasi 2007: 324).

En el huerto se entiende a la naturaleza como sustancia de una industria. El jardín que crea Charlotte sigue, en cambio, criterios sentimentales. Pero ambas son formas estéticas de relación con la naturaleza, en tanto que son dos maneras de percepción de la misma. Formas en cierta medida contrapuestas: mientras que la primera contempla la naturaleza según el criterio de utilidad, la segunda según el criterio sentimental de belleza; mientras que la primera pone el acento en el proceso ${ }^{5}$, la

\footnotetext{
${ }^{4}$ Nota aclaratoria: Los pasajes de Die Wahlverwandtschaften irán acompañados de la indicación bibliográfica correspondiente a su decimocuarta edición en el Deutscher Taschenbuch Verlag. A la hora de citar pasajes del resto la obra de Goethe no tomados de obras que los citen, seguimos la notación canónica propuesta por la Goethe-Gesellschaft. Como nos basamos principalmente en la Münchner Ausgabe, citaremos siempre en esos casos con MA seguido del número del volumen y el número de página(s). Sólo habrá dos casos en los que citemos un pasaje tomado de la edición de Frankfurt: la cita estará entonces precedida de las siglas FA.

${ }^{5}$ Este modo de percibir la relación entre naturaleza y arte es de raigambre winckelmanniana. A propósito de la teoría de Winckelmann, Ponzi escribe (PONZI 2007: 14): "Il paradosso consiste nel fatto che una teoria, dall'impronta idealistica e neoplatonica così marcata, formula per prima il
} 
segunda lo hace en la imagen. Pero ambas son formas de ejecución de un juicio sobre el medio natural, en base al cual lo transforman, siguiendo sus propios principios. Dos estéticas, por tanto, autónomas la una de la otra, aunque vinculadas entre ellas. Relación ambigua simbolizada en Die Wahlverwandtschaften por la separación vinculante del muro imaginario que distingue ambas partes del jardín en torno al huerto y que continúan la línea, por ejemplo, de la oposición entre vita activa y vita contemplativa del Torquato Tasso ${ }^{6}$-pieza teatral que, no en vano, discurre en los dominios (jardín incluido) del castillo de Belriguardo-: como lo mostraremos más abajo, Charlotte será aquí la representante de un modo éticamente contemplativo de abordar la naturaleza; Eduard lo hará, en cambio, de forma patéticamente activa.

Pero damos un paso más: las dos estéticas son económicas, entendida la economía, de forma política, como un sistema de ordenación (Vogl 2008: 11 y Foucault 2004). Dos formas, en definitiva, de organización racional del espacio, herederas a su modo de la economía política que se viene articulando desde finales del siglo XVII, produciendo un profundo cambio epistémico en un proceso que concluirá en torno a 1800 :

Diese politische Ökonomie hat sich [...] nicht nur als ein neuer Gegenstandsbereich vorgestellt (und sich etwa gegen die Prinzipien der alten, aristotelischen Ökonomik ebenso abgesetzt wie gegen die der Staatsräson), sondern als ein weitläufiger Diskurszusammenhang, der eine zentrale Position in der Kultur der Aufklärung besetzt und politische, anthropologische, sozialphilosophische und ästhetische Dimensionen gleichermaßen umfasst. Um 1800 schließlich [...] hat sich dieses ökonomische Wissen wiederum neu konstelliert. Das betrifft nicht nur den Zerfall kameralistischer Ordnungsversuche; das betrifft nicht nur die Kritik, die etwa die physiokratischen Theorien der Wertbildung erfahren. Und das betrifft auch nicht allein die Entstehung eines eigenständigen nationalökonomischen Fachs, das sich aus der Gesamtheit des Staats- und Regierungswissens emanzipiert und nun den autonomen Gesetzmäßigkeiten eines wirtschaftlichen Funktionsystems zu verschreiben versucht. Es lässt sich um und nach 1800 vielmehr ein grundlegender Wechsel der Kategorien feststellen, mit denen man die ökonomischen Kräfte, die umlaufenden Reichtümer und die Gesetze ihrer Herstellung analysiert (Vogl 2008: 12).

Die Wahlverwandtschaften se sitúa precisamente en este tiempo liminar: la economía de esta novela publicada en 1809 sigue unas determinadas reglas de

procedimento dell'arte come "costruzione" secondo un progetto estetico ben preciso che non è dissimile dalla progettualità e dal calcolo della concezione artistica delle avanguardie. Il "bello ideale" è definito mediante un procedimento : ossia indicando le procedure per la sua rappresentazione, ma la "bellezza in sé" non è definibile." El modo en que Eduard percibe la naturaleza le debe mucho a este modo estético de abordarla: constantemente en la novela se aprecian huellas del modo en que tiene de percibir lo natural como proceso; algunas de ellas serán reflejadas en este artículo.

${ }^{6}$ En este sentido juzga, por ejemplo, Alfons a Tasso: “ALFONS. Es ist ein alter Fehler, dass er mehr / Die Einsamkeit als die Gesellschaft sucht” (MA 6, 220, v. 244s). 
organización representadas a lo largo de la diégesis y que simbólicamente $e^{7}$ pueden caracterizarse recurriendo al injerto, el correlato botánico de las afinidades electivas, y a la ventana. Tanto el uno como la otra son dos formas de acercamiento a la naturaleza que la novela combina y que están representadas, respectivamente y con sus gradaciones, por Eduard y el mayor por un lado, y por Charlotte y Ottilie, por otro. Y este hecho no es una casualidad, sino que obedece a una forma de organización poetológica determinada por principios y conclusiones a los que Goethe ha ido llegando en el curso de sus estudios tan científicos como económicos, en el sentido, repetimos, de sistema de organización de la realidad natural, en nuestro caso-. Die Wahlverwandtschaften es una novela que, a su modo, presenta un doble acercamiento crítico a dos formas distintas de abordar el medio en el siglo XVIII: la micrológica del injerto y la juntura; la macrológica de la ventana y la panorámica.

Estas dos maneras de abordar el medio natural las combina también en su historia sobre la "Pflanzencultur" en el gran principado de Weimar. Cuando se refiere a los primeros trabajos de ajardinamiento a la inglesa del Ilmtal, escribe:

Im ganzen ist man überall bemüht der Örtlichkeit ihr Recht widerfahren zu lassen, sie möglichst zu benutzen und nichts gegen ihren Charakter zu verfügen.

Im ernstlichen geht die regelmäßige Forstkultur im Lande fort, damit verbindet sich schon die Erziehung fremder Baumarten.

Große Anpflanzungen und sonstiges Vermehren geschieht durch einsichtige Forstmänner; dadurch gewinnt man an Erfahrung welche Pflanzen unser Klima ertragen können.

Hier wäre etwas Näheres über die rauhere Lage von Weimar und Belvedere zu sagen; unsere Höhe ist schon bedeutend, die Nähe vom Thüringer Wald und zwar die Lage desselben in Süden hat nicht weniger Einfluß; die nordöstlichen und nordwestlichen Zugwinde bedrohen die Vegetation gar öfters (FA 24, 539).

Vemos combinadas en este fragmento del esquema para la historia de la botánica en Weimar las dos formas de acercamiento a lo natural que también encontramos en la novela. Lo interesante de este pasaje es, además, el modo en que se encadenan las ideas: el experimento a nivel micrológico de plantación de especies no autóctonas permite saber cuáles soportan el clima de Weimar, lo que a su vez lleva a convertir en pertinente una reflexión general a nivel climático sobre la situación geográfica de Weimar. Un ejemplo más de cómo el jardín y las actividades en él realizadas contribuyen al conocimiento -en este caso climático, geográfico y botánico- ${ }^{8}$.

${ }^{7}$ Friedrich Wilhelm RIEMER, Tagebuch vom 28. August 1808: "Goethes Geburtstag. Mit ihm über den neueren Roman, besonders den seinigen".

"Er äußerte, seine Idee bei dem neuen Roman "Die Wahlverwandtschaften" sei: sociale Verhältnisse und die Conflicte derselben symbolisch gefaßt darzustellen" (GOETHE 2009: 259).

${ }^{8}$ De forma paralela anota Goethe además la ampliación de la biblioteca botánica, la adquisición de nuevas y raras especies de plantas, y la construcción de instalaciones adecuadas para la mejora y 
"Jede Wissensordnung [bildet] bestimmte Darstellungsoptionen [aus]" (Vog1 2008: 13). Este es el principio básico del que también partimos nosotros en este artículo y según el que, en nuestra opinión, puede entenderse en toda su intensidad lo enraizado que está el marco simbólico que le da el jardín a la novela en la situación estética, científica y económica de la época.

Lo característico de la presentación simultánea y no orgánicamente integrada que hace Goethe de estas visiones de lo natural, es que su combinación obedezca a un objetivo que comparten: para cuando se publica la novela, estas formas de abordar lo natural han sido objeto de crítica por parte de Goethe. La forma en que tanto el mayor como el barón ven la naturaleza tiene bastantes elementos en común con la figura del Charakteristiker, del que en Der Sammler und die Seinigen se dice:

Er glaubte man könne sie auch in einem gewissen Sinne Rigoristen nennen. Ihre Abstraktion, ihre Reduktion auf Begriffe begründe immer etwas, führe zu etwas, und gegen die Leerheit anderer Künstler und Kunstfreunde gehalten, sei der Charakteristiker besonders schätzbar.

Der kleine, hartnäckige Philosoph aber zeigte auch hier wieder seinen Zahn, und behauptete: daß ihre Einseitigkeit, eben wegen ihres scheinbaren Rechtes, durch Beschränkung der Kunst weit mehr schade als das Hinausstreben des Imaginanten (MA 13, 314-315).

Y el modo en que Charlotte transforma y concibe el medio natural lo rechaza Goethe porque disuelve lo natural en lo artístico (Träger 1999: 124), algo que ya había criticado en 1799 en su esquema sobre el diletantismo (cit. ibíd.): "Vorliebnehmen mit dem Schein. Vermischung von Kunst und Natur" y que se encuentra estrechamente vinculado al tipo de los Imaginanten:

Man nannte sie Poetisierer, weil sie, anstatt den poetischen Teil der bildenden Kunst zu kennen und sich darnach zu bestreben, vielmehr mit dem Dichter wetteifern, den Vorzügen desselben nachjagen und ihre eignen Vorteile verkennen und versäumen. Man nannte sie Scheinmänner, weil sie so gern dem Scheine nachstreben, der Einbildungskraft etwas vorspielen suchen, ohne sich zu bekümmern inwiefern dem Anschauen genug geschieht. Sie wurden Phantomisten genannt, weil ein hohles Gespensterwesen sie anzieht, Phantasmisten, weil traumartige Verzerrungen und Inkohärenzen nicht ausbleiben, Nebulisten, weil sie der Wolken nicht entbehren könne, um ihren Luftbilder einen würdigen Boden zu verschaffen.

mantenimiento del complejo (FA 25, 540). Esta serie de actividades son también una forma de concretar las diferentes formas de creación, archivo y proyección del conocimiento a la que hemos hecho referencia en el epígrafe introductorio. 
Ambos tipos no sólo están predestinados por una mano invisible a separarse, sino que aportan con sus modos de ver, interpretar y proyectar la realidad natural acción al paisaje, ayudados, una vez transcurra un poco más de tiempo diegético en la novela, por las dos figuras que ampliarán la vida en pareja inicial entre Eduard y Charlotte.

\section{Tiempo y naturaleza: paseos por el Faust y por Die Wahlverwandtschaften}

En su artículo Formen der Verzeitlichung, Matussek (1998) resume las conclusiones de su habilitación sobre la caracterización del Faust como un "Drama der Verzeitlichung" (Matussek 1998: 202). Una hipótesis que desarrolla analizando cuatro monólogos del Fausto, escritos en diferentes etapas creativas. Uno de ellos es el pronunciado en la escena conocida como el Osterspaziergang (MA 5, 171-172, vv. 903-940), que fue concebido en 1808. Es decir, en medio del proceso de ampliación de la Novelle sobre las afinidades electivas en el marco de los Wanderjahre -a partir de 1807- y la publicación de la novela Die Wahlverwandtschaften a principios de 1809 (Bolz 1997: 153). De esta estrecha vecindad temporal partimos para establecer relaciones analógicas entre lo afirmado por Matussek para el caso del discurso de Fausto en el Osterspaziergang y una serie de pasajes de la novela que nos ocupa. Las observaciones de Matussek sobre el discurso son las siguientes (1998: 219ss):

- Se trata del primer discurso sostenido por Fausto fuera de su cuarto de estudio.

- En él se reproducen panorámicas contempladas por Fausto y Wagner a vista de pájaro desde un promontorio y en las que reflejan la eclosión primaveral de la naturaleza y el ocio de los burgueses de forma global y no secuencialmente hipertextualmente deduciríamos-: "Dadurch erst kann das Einzelne zum Repräsentanten des Ganzen werden. Die Synekdoche findet stets einen totalisierenden kategorialen Rahmen" (Matussek 1998: 220).

- Los procesos naturales que se registran se corresponden con las actividades festivas de los hombres, con lo que la historia humana y la natural se presentan como estrechamente relacionadas (Matussek 1998: 220):

Der Bildungsvorgang der Natur ergibt sich aus dem Widerstreit zwischen winterlicher Erstarrung und frühlingshaftem Aufbruch, kontrahierender Schneedecke und expandierendem Sonnenlicht. Dieser Vorgang wiederum spiegelt sich, mit derselben kontrapunktistischen Struktur, im Bereich der Gesellschaft: Auch die Bürger der Stadt stehen in der Polarität zwischen "Gewerbesbanden" und Freizeit, strömen aus der bedrückenden "Enge" der Stadt ins Weite der Landschaft, aus dem "finstren Tor" “ans Licht" des sonnigen Sonntags ([MA 5, 172,] V. 924-928).

- El simbolismo del Osterspaziergang sigue, por tanto, las máximas estéticas que Goethe había desarrollado durante su trabajo con Schiller -sobre todo en la 
publicación Propyläen- (Matussek 1998: 221). Fausto tiene la misma mirada idealizante que reclamaba Goethe para el artista en la introducción a las Propyläen:

Indem der Künstler irgendeinen Gegenstand der Natur ergreift, so gehört dieser nicht mehr der Natur an, ja man kann sagen: daß der Künstler ihn in diesem Augenblick erschaffe, indem er ihm das Bedeutende, Charakteristische, Interessante abgewinnt oder vielmehr erst den höheren Wert hineinlegt (MA 13, 145).

- Sin esta mirada idealizante no podría ni elevar los fenómenos naturales ni desligar las actividades de los hombres de su contingencia histórica. Este proceso idealizante lo definió Goethe sobre todo en el Symbolbrief, la conocida carta que dirigió a Schiller desde Frankfurt el 16 de agosto de 1797, y en el que afirmaba la existencia de "eminente Fälle" que, funcionando a modo de objetos simbólicos, actuaban como representantes de muchos otros casos, encerrando en ellos una cierta totalidad que los hace abrirse a un "Anschauen" sensorial (cit. Matussek 1998: 221).

- Por todo ello, en la Szenerie del monólogo hay una relación orgánica entre las esferas del paisaje y de las festividades de los hombres (Matussek 1998: 221).

Esta elaboración compositiva entre fenómenos naturales y acontecimientos sociales que analiza Matussek, se fundamenta en su opinión en tres pilares (1998: 222):

1) La filosofía trascendental de Kant, que permite la vinculación de observaciones científicas de manera que permitan deducir de las mismas principios racionales.

2) El dualismo filosófico-reflexivo de Schelling y el concepto de identidad a él ligado:

Die Natur soll das sichtbare Geist, der Geist die unsichtbare Natur seyn. Hier also, in der absoluten Identität des Geistes in uns und der Natur außer uns, muß sich das Problem, wie eine Natur außer uns möglich sey, auflösen (Matussek 1998: 224).

En él funda Goethe su concepción objetivo-idealista de las dos Triebräder aller Natur: el principio de polaridad y el de la Steigerung, a partir de los que concibe un "Modell einer Steigerung aus Polaritäten" (cit. Matussek 1998: 225).

3) La concepción armonizante de la naturaleza que desarrolla en el principio de metamorfosis de las plantas y por el que coloca a la "Natur im Zeichen der Geschichte" (Matussek 1998: 226).

Todas estas observaciones que hace Matussek a propósito del monólogo de Fausto en el Osterspaziergang son extrapolables a la novela Die Wahlverwandtschaften. En ella la relación entre la acción en torno a los personajes y las transformaciones que sufre el jardín -cíclicas o provocadas por el hombreobedece a la figura de la sinécdoque: cada acción del medio o de los personajes son parte de un todo que resulta de desligar lo anecdótico de su carácter contingente y elevarlo a la categoría de lo simbólico, resumida en la denominación del fenómeno químico que da título a la novela. Los movimientos básicos que 
caracterizan la relación tanto entre los personajes como entre las partes del jardín entre sí, como de éstas con los personajes son, como en el monólogo del Fausto, la sístole y la diástole. Así, las estaciones del año que tienen especial protagonismo en la novela serán la primavera -estación de la expansión de las plantas- y el otoño - estación de la contracción de la naturaleza preparatoria para el invierno y que en la novela se destaca por ser el momento en que las flores pasan a ser frutos-; hay momentos de la novela caracterizados por la ausencia del mayor y del barón, cuya presencia en la trama se contrae, por tanto, al tiempo que adquiere más protagonismo -se expande- la del arquitecto; o, como último ejemplo, la expansión del jardín inglés desarrollado por Charlotte está íntimamente relacionada con la contracción, con el cierre de las tumbas del cementerio y la reorganización de la distribución de los monumentos que lo forman.

Asimismo, es una constante en la novela que se desliguen acontecimientos puramente anecdóticos para convertirlos en casos eminentes ${ }^{9}$, siguiendo el planteamiento del Symbolbrief. Así, por ejemplo, como veremos más abajo, el cumpleaños de Ottilie y el día en que Eduard plantó unos plátanos junto a uno de los lagos se desligan de su carácter contingente en el momento en que Eduard, al revisar los diarios de su padre, cae en la cuenta de que ambas fechas coinciden: desde ese momento, desde que se da cuenta de la vinculación, no las ve como algo casual, sino que las dota patéticamente de significado.

En resumen, la forma en que Fausto observa la realidad natural y la social como vinculadas es muy similar a la estructura de muchos de los pasajes de Die Wahlverwandtschaften, como, por ejemplo, cuando, nada más comenzar la narración, el barón Eduard es acomodado por su mujer Charlotte en la cabaña de musgo de manera que pueda observar a través de la ventana el paisaje como si éste fuera un cuadro o la reproducción viva del mismo:

An der Tür empfing Charlotte ihren Gemahl und ließ ihn dergestalt niedersitzen, daß er durch Tür und Fenster die verschiedenen Bilder, welche die Landschaft gleichsam im Rahmen zeigten, auf einen Blick übersehen konnte. Er freute sich daran in Hoffnung, daß der Frühling bald alles noch reichlicher beleben würde (Goethe 2009: 8).

Tiempo y naturaleza están íntima, en tanto que simbólicamente, relacionados en el espectáculo del jardín de Die Wahlverwandtschaften. El barón espera, cuando ve el espectáculo de la naturaleza enmarcado en la ventana, que la primavera enriquezca todo pronto. El acercamiento a la naturaleza como imagen, puesto de manifiesto por los esfuerzos de Charlotte por ubicar correctamente a su marido para que vea convenientemente el espectáculo, se combina con la visión prácticamente proyectista del barón, que espera, imagina. Mientras que Charlotte

\footnotetext{
${ }^{9}$ La inclusión de este tipo de casos refleja en cierto modo la intrínseca síntesis entre destrucción y orden (ZABKA 1998: 161) que Goethe hace en el Symbolbrief cuando reflexiona sobre el carácter industrioso de los habitantes de Frankfurt a pesar de la destrucción que traen las guerras napoleónicas. Desgraciadamente no podemos insistir en este extremo.
} 
presenta la naturaleza, el barón piensa en su futuro. Pero ambos modos de ver ponen en evidencia una estrecha vinculación entre tiempo y naturaleza. Relación en la que abundaremos en el siguiente epígrafe.

\section{Tiempo, naturaleza, historia, diégesis}

En su obra Kalkül und Leidenschaft, Joseph Vogl analiza la relación entre literatura y economía desde una perspectiva poetológica. Literatura y economía son dos formas de conocimiento que implican un modo determinado de organización del discurso (Vogl 2008: 14). En el caso de Die Wahlverwandtschaften esta relación se concreta en el fenómeno del tiempo, hasta el punto de que se puede resumir la estructura poetológica de la novela en una combinación de tres elementos, que concreta al tratar el capítulo cuarto, en el que Otto, el Hauptmann, les habla al barón, a Charlotte y a Ottilie de la teoría de las afinidades electivas, de la Gleichnisrede:

Der Gegenstand der Gleichnisrede - das Verhältnis von Wissen und Leben - ist bereits in deren Bedingungen akut, als Problem von Wissen und Leben. Gleichnishaftigkeit einerseits und die Verzeitlichung, d.h. die Modernisierung des Wissen andererseits sind auf intrinkate, zirkuläre Weise miteinander verzahnt. Das Gesetz der gleichnishaften Übertragung und das Gesetz, das im Gleichnis übertragen wird, präsentieren sich als die beiden Enden derselben Ungewissheit, einer Ungewissheit, in der die Wahrheit als Verzeitlichung und eine verzeitlichte Wahrheit einander berühren. Dieser Zirkel ist es, der - spielerisch eingeführt - zum Ernstfall der Figuren gerät; und dieser Zirkel ist es, an dem sich Goethes Roman als Literatur einer Zeitenwende und als eine eminente Frage des Wissens definiert: als eine Frage, die sich im Dreieck zwischen Gesetzmäßigkeit (die Anziehungen und Abstoßungen), ihrer Repräsentation (in Zeichen und Deutungen) und der Zeit (als Gegenstand und Bedingung des Wissens) enfaltet (Vogl 2008: 292).

El tiempo, como lo hemos visto ya desde una perspectiva filosófica de la mano de Matussek, es el que articula a nivel simbólico la novela. Pero si añadimos el punto de vista de Vogl, vemos que el jardín tiene su protagonismo y su función poetológica - en íntima relación con el conocimiento- precisamente de la mano del tiempo. A medida que las reformas en los jardines se van realizando, el conocimiento sobre la distribución y composición del terreno por parte de los protagonistas $-\mathrm{y}$, por ende, del lector- va aumentando. Este conocimiento tiene al menos dos consecuencias importantes: primero, que el lector va conociendo cada vez más a los personajes, que se autodefinen por su forma de percibir el paisaje y concebir posibles cambios en el mismo ${ }^{10}$; y segundo, que el proceso de

${ }^{10}$ Esta forma de conocimiento es la que vincula Landschaftsgarten y Bildungsreise en la reflexión autobiográfica de Goethe (TRÄGER 1999: 125). 
conocimiento más exacto del jardín desencadena -o acompaña- cambios en las relaciones entre las figuras.

De esta manera, el tiempo cíclico del calendario estacional al que nos hemos referido en el apartado anterior aparece como una de las múltiples caras en las que se concreta el tiempo en el fenómeno cambiante del jardín. Al tiempo histórico -sea calendario, agenda o diario- se le une forzosamente el tiempo diegético: el jardín va transformándose a medida que van mutando las relaciones entre los cuatro personajes principales de la novela.

Dos de los momentos en el que la relación entre diégesis, autodefinición del personaje y tiempo histórico-cíclico es especialmente intensa están colocados sintomática e intencionadamente al principio de cada una de las dos partes en que se divide la novela.

El primero es el pasaje al que ya nos referimos en el segundo epígrafe y en el que coinciden la primavera, la conclusión de dos obras por parte de Eduard y Charlotte -el injerto y la construcción y acondicionamiento de la cabaña de musgo, respectivamente- que definen su relación con el huerto y con el jardín inglés, y el estadio inicial de la diégesis de la novela.

El segundo es el pasaje que se inicia con la llegada de un joven abogado al dominio del barón, cuando éste y el mayor están ausentes. El narrador interviene en la diégesis para subrayar la importancia de este episodio mediante la irónica afirmación: "Wir müssen dieses Vorfalls gedenken, weil er verschiedenen Dingen einen Anstoß gab, die sonst vielleicht lange geruht hätten" (Goethe 2009: 125-126). A continuación hace una breve crónica de las reformas que Charlotte había realizado en el cementerio, colocando las lápidas y los monumentos alineados contra la pared, aplanando el terreno y sembrándolo de trébol, de manera que el recuerdo de los muertos queda desligado de la ubicación exacta de sus restos por conferir al lugar una belleza uniformizante. Ésta es la causa del problema que plantea el abogado: dado que la nueva distribución de los monumentos no permite localizar el lugar donde descansan los restos de los enterrados allí, su cliente se ve legitimado para cancelar la obligación de pagar una renta a cambio de que los cuerpos de sus antepasados descansen en el cementerio. Después de aducir sus argumentos, el abogado concluye:

Ich halte deswegen dafür, daß mein Prinzipal völlig recht habe, die Stiftung zurückzunehmen; und dies ist noch billig genug, denn die Glieder der Familie sind auf eine Weise verletzt, wofür gar kein Einsatz zu denken ist. Sie sollen das schmerzliche süße Gefühl entbehren, ihren Geliebten ein Totenopfer zu bringen, die tröstliche Hoffnung, dereinst unmittelbar neben ihnen zu ruhen (Goethe 2009: 127-128).

A ello responde Charlotte:

Ihre Argumente haben mich nicht überzeugt. Das reine Gefühl einer endlichen allgemeinen Gleichheit, wenigstens nach dem Tode, scheint mir beruhingender als dieses eigensinnige, starre Fortsetzen unserer Persönlichkeiten, Anhänglichkeiten und Lebensverhältnisse. - Und was sagen Sie hierzu? (Goethe 2009: 128). 
Pregunta, dirigiéndose al arquitecto, que responde:

Ich möchte [...] in einer solchen Sache weder streiten noch den Ausschlag geben. Lassen Sie mich das, was meiner Kunst, meiner Denkweise am nächsten liegt, bescheidentlich zu äußern. [...] Wenn die Glieder einer Gemeinde reihenweise nebeneinander liegen, so ruhen sie bei und unter den Ihrigen; und wenn die Erde uns einmal aufnehmen soll, so finde ich nichts natürlicher und reinlicher, als daß man die zufällig entstandenen, nach und nach zusammensinkenden Hügel ungesäumt vergleiche und so die Decke, indem alle sie tragen, einem jeden leichter gemacht werde (Goethe 2009: 128).

En este momento interviene Ottilie: "Und ohne irgendein Zeichen des Andenkens, ohne irgend etwas, das Erinnerung entgegenkäme, sollte das alles so vorübergehen?" (Goethe 2009: 128). Y el arquitecto replica:

Keineswegs! [...], nicht vom Andenken, nur vom Platze soll man sich lossagen. Der Baukünstler, der Bildhauer sind höchlich interessiert, daß der Mensch von ihnen, von ihrer Kunst, von ihrer Hand eine Dauer seines Daseins erwarte; und deswegen wünschte ich gut gedachte, gut ausgeführte Monumente, nicht einzeln und zufällig ausgesäet, sondern an einem Orte aufgestellt, wo sich Dauer versprechen können. [...] (2009: 128-129).

Charlotte interviene preguntándole al arquitecto: si los artistas son tan imaginativos, ¿por qué se repiten en la mayoría de las veces los motivos de las losas? Y aquél responde:

Das ist wohl bei uns so [...], aber nicht überall. [...] Was Entwürfe zu Monumenten aller Art betrifft, deren habe ich viele gesammelt und zeige sie gelegentlich; doch bleibt immer das schönste Denkmal des Menschen eigenes Bildnis. Dieses gibt mehr als irgend etwas anders einen Begriff von dem, was er war; es ist der beste Text zu vielen oder wenigen Noten; nur müsste es aber auch in seiner besten Zeit gemacht sein, welches gewöhnlich versäumt wird. Niemand denkt daran, lebende Formen zu erhalten, und wenn es geschieht, so geschieht es auf unzulängliche Weise. Da wird ein Toter geschwind noch abgegossen und eine solche Maske auf einen Block gesetzt, und das heißt man eine Büste. Wie selten ist der Künstler imstande, sie völlig wiederzubeleben! (Goethe 2009: 129).

\section{A lo que replica Charlotte triunfante:}

Sie haben, ohne es vielleicht zu wissen und zu wollen [...] dies Gespräch ganz zu meinen Gunsten gelenkt. Das Bild eines Menschen ist doch wohl unabhängig; überall, wo es steht, steht es für sich, und wir werden von ihm nicht verlangen, daß es die eigentliche Grabstätte bezeichne. Aber soll ich Ihnen eine wunderliche Empfindung bekennen? Selbst gegen Bildnisse habe ich eine Art von Abneigung; denn sie scheinen mir immer einen stillen Vorwurf zu machen [...]. Ich hörte fragen, warum man von den Toten so unbewunden Gutes sage, von Lebenden immer mit einer ge- 
wissen Vorsicht. Es wurde geantwortet: weil wir von jenen nichts zu befürchten haben und diese uns noch irgendwo auf den Weg kommen könnten. So unrein ist die Sorge für das Andenken der andern (Goethe 2009: 129-130).

Hemos citado amplios pasajes de este diálogo a cuatro para mostrar de forma evidente la íntima relación que tiene con la famosa conversación sobre las afinidades electivas del capítulo cuarto. La reflexión que se desata por una disputa legal, producida por las reformas en el cementerio que hizo Charlotte, concluye de una manera análoga a la Gleichnisrede: el subtexto de Die Wahlverwandtschaften es el estado de intercambiabilidad de los elementos de una relación cuando se dan ciertas circunstancias-marco ${ }^{11}$. Así, en la teoría química es la aparición de un nuevo elemento lo que provoca que la atracción previa entre dos naturalezas se elimine por la fuerza que el nuevo elemento ejerce sobre una de las naturalezas dadas. En el caso del diálogo sobre el asunto de los monumentos del cementerio, el esquema se repite: el nuevo elemento, esto es la nueva disposición de los monumentos por las reformas de Charlotte, hace que la íntima relación entre losa y restos se disuelva y en su lugar surjan otras dos: la de los restos entre sí, al "soportar juntos" la misma tierra, y la de la imagen que recuerda al finado y el cuerpo vivo de éste. El desarrollo del diálogo pone de manifiesto el carácter contingente de la relación directa entre monumento y lugar donde descansan los restos del recordado, al abrir la posibilidad de que se dé otra relación ya no tradicional, sino estética entre monumento y finado.

El subtexto que Goethe utiliza en la descripción de esta relación estética lo constituyen sus propias ideas sobre las obras de arte vivas (Träger 1999 133-135) y que será utilizado varias veces a lo largo de la novela, por ejemplo ya hacia el final se dice del arquitecto, cuando éste visita la tumba de Ottilie, que "schon einmal hatte er so vor Belisar gestanden" (Goethe 2009: 252), haciendo referencia directa al grabado de un cuadro de Van Dyck que tenía el arquitecto y que había mostrado en sociedad capítulos atrás. Goethe prefiere la obra de arte viva a la muerta, tal y como se desprende tanto de las Weimarer Preisausschreibungen y de sus reflexiones para las Propyläen (Träger 1999: 138). Así, se puede sostener que quizá toda la novela no sea otra cosa que un intento de presentar artísticamente vivos a dos personajes desde un principio muertos o al menos llamados a morir: Eduard y Ottilie. Lo que potenciaría el carácter profundamente trágico de Die Wahlverwandtschaften.

Si resumimos lo dicho y lo transcrito de este fragmento estableciendo una relación con lo afirmado a propósito del pasaje que abre la novela, vemos que el diálogo tiene lugar en un tiempo histórico-biográfico determinado por la ausencia de Eduard y del Mayor, después de que Charlotte haya reorganizado el cementerio y en la mitad de la diégesis de la novela. Esta triple circunstancia, unida a la aparición de

\footnotetext{
${ }^{11}$ De forma análoga a como ocurre con la relación entre las artes en la concepción estética del último Goethe. Como lo recoge Träger a propósito de una conversación de Goethe con Eckermann sobre las ilustraciones de Delacroix al Fausto (TRÄGER 1999: 137): “Auf dem gemeinsamen figuralen Nenner ließen sich Plastik, Malerei, Literatur, Schauspiel und lebendiges Dasein in einen Zustand der Austauschbarkeit versetzen. Allein der Grad der Stilisierung hob die Gebilde der Kunst über die Zufälligkeit des Lebens hinaus".
} 
un tercer personaje que, como se dice explícitamente ${ }^{12}$, suple la ausencia de Eduard y Otto, es la que da pie a un debate en el que Ottilie y Charlotte se pueden caracterizar a sí mismas como dos personajes en cuyo modo de hacer predominan las características de la vita contemplativa, pero con un matiz diferenciador: la argumentación de Charlotte, una mujer madura y educada, obedece a un ethos bien marcado sobre todo en su última intervención, que cierra brillantemente el diálogo; Ottilie sólo interviene una vez, interrumpiendo al arquitecto por no poder aceptar desde su pathos la idea de que no haya posibilidad de vincular recuerdo y cuerpo sepultado, como si todo ocurriera para nada.

En este sentido, observando con atención las acciones de los personajes a propósito de la reforma del jardín, puede deducirse que la relación entre ellos se puede establecer en base a sus concepciones vitales, puestas precisamente de manifiesto en su relación con el jardín. Como no podemos probar punto por punto este aspecto lo resumimos en el siguiente esquema (Fig. 1):

Eduard Charlotte

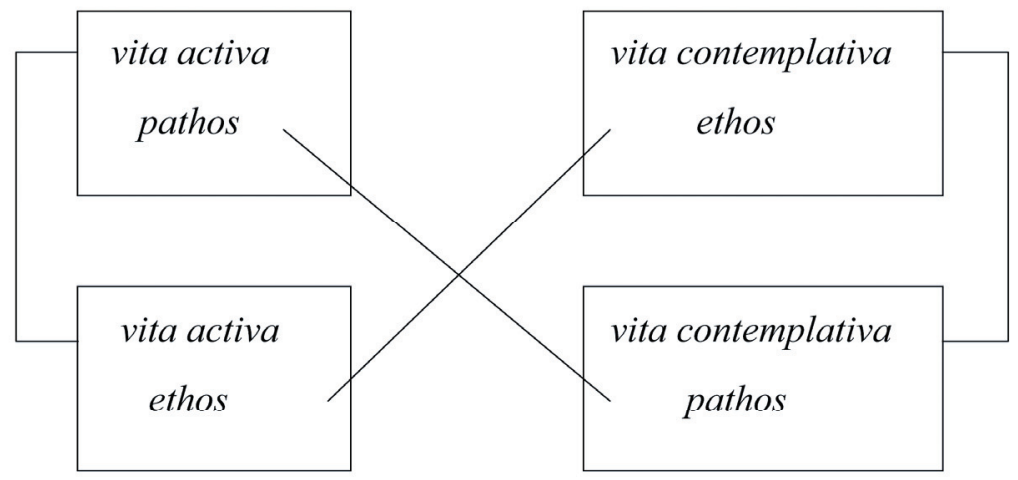

Otto Ottilie

Fig. 1: Relación entre los personajes

\footnotetext{
12 "Im gemeinen Leben begegnet uns oft, was wir in der Epopöe als Kunstgriff des Dichters zu rühmen pflegen, daß nämlich, wenn die Hauptfiguren sich entfernen, verbergen, sich der Untätigkeit hingeben, gleich sodann schon ein Zweiter, Dritter, bisher kaum Bemerkter den Platz füllt und, indem er seine ganze Tätigkeit äußert, uns gleichfalls der Aufmerksamkeit, der Teilnahme, ja des Lobes und Preises würdig erscheint.

So zeigte sich gleich nach Entfernung des Hauptmanns und Eduards jener Architekt täglich bedeutender, von welchem de Anordnung und Ausführung so machen Unternehmens allein abhing, wobei er sich genau, verständig und tätig erwies und zugleich den Damen auf mancherlei Art beistand und in stillen, langwierigen Stunden sie zu unterhalten wußte" (GOETHE 2009: 123).
} 
Los cuatro personajes están vinculados y se diferencian entre ellos por relaciones de afinidad positiva o negativa (magnética). El mayor y el barón sobre hombres de vita activa -como Alfons en el Torquato Tasso-, mientras que las mujeres son representantes de la vita contemplativa del propio Tasso. Pero cada uno a su manera, y ahí está la diferencia sutil entre esas dos parejas de personajes: el mayor y Charlotte son figuras en las que predomina el carácter ético, mientras que el barón y Ottilie son personajes patéticos.

Con este esquema delante, se puede entender la opinión de Goethe sobre las críticas a su novela que recoge Riemer:

Gespräch mit Riemer. Weimar, Dezember 1809

Unter andern Philister-Kritiken über die "Wahlverwandtschaften", gleich nach ihrer Erscheinung, war auch die: daß man keinen Kampf des Sittlichen mit der Neigung sehe. - Goethe bemerkte dabei gegen mich: "Dieser Kampf ist aber hinter die Scene verlegt, und man sieht, daß er vorgegangen sein müsse. Die Menschen betragen sich wie vornehme Leute, die bei allem innern Zwiespalt doch das äußere Decorum behaupten. Der Kampf des Sittlichen eignet sich niemals zu einer ästhetischen Darstellung: denn entweder siegt das Sittlicher oder es wird überwunden. Im ersten Fall weiß man nicht, was und warum es dargestellt worden; im andern ist es schmächlich, das mitanzusehen. Denn am Ende muß doch irgend ein Moment dem Sinnlichen das Übergewicht geben, und dieses Moment gibt der Zuschauer gerade nicht $\mathrm{zu}$, sondern verlangt ein noch schlagenderes, das der Dritte immer wieder eludiert, je sittlicher er selbst ist. In solchen Darstellungen muß stets das Sinnlich Herr werden, aber bestraft durch das Schicksal, das heißt: durch die sittliche Natur, die sich durch den Tod ihre Freiheit salviert. So muß Werther sich erschießen, nachdem er die Sinnlichkeit Herr über sich selber lassen; so muß Ottilie karterieren und Eduard desgleichen, nachdem sie ihrer Neigung freien Lauf gelassen. Nun feiert erst das Sittliche seinen Triumph" (Goethe 2009: 260-261).

Die Wahlverwandtschaften es una colección de personajes que portan una serie de máscaras convencionales que irán destapándose a medida que avance la diégesis y el lector vaya conociendo mejor a los protagonistas. En éste proceso ve Goethe la garantía de que no se pone en cuestión ninguna ley social.

\section{Coleccionistas: tiempo, acumulación y la estética de la obra de arte viva}

Otra función poetológica del tiempo es la recurrente aparición del tema de la colección o del archivo en la novela. Especialmente en relación con el jardín: son muchos los personajes de la novela que coleccionan objetos destinados a la observación del espacio. Desde la colección de grabados del arquitecto, pasando por los instrumentos de medición del mayor hasta llegar a la camara obscura del inglés y su acompañante, todos ellos son testimonio de una relación cambiante con el medio físico que se traduce en nuevas formas de observación y percepción del mismo. 
En el caso del jardín, se produce una doble influencia de la colección o el archivo, dado que él mismo es archivo y colección de modificaciones hechas al terreno con fines industriales en el caso del huerto o puramente estéticos en el caso del jardín inglés. Incluso la propia existencia del jardín es objeto de historia, por ejemplo en forma escrita en el caso de los diarios del padre de Eduard o de la propia Ottilie o en forma oral en la memoria del viejo jardinero. De esta manera se constata una íntima relación entre tiempo y espacio desde una perspectiva distinta, hipertextual, concretada especialmente en la acumulación de distintos puntos de vista sobre el jardín con la que se va estructurando la diégesis de la novela: desde la ventana de la cabaña de musgo de Charlotte, pasando por el mapa topográfico que hace el mayor de los terrenos del barón, hasta las imágenes de la camara obscura del inglés y su acompañante, la imagen del jardín se multiplica y se dinamiza pasando de la mera contemplación estética a la observación precisa y realista. Simultáneamente se constata con cada nueva imagen que las anteriores son pasado, porque entre tanto se han introducido cambios y reformas en los terrenos que circundan el castillo del barón. Y la colección de imágenes e impresiones de los protagonistas en la que se convierte la novela recoge las huellas de este cambio y lo hacen visible, en tanto que vivo, íntimamente en consonancia con la defensa de la obra de arte viva que hace Goethe teórica y políticamente a partir de 1800 .

Veamos dos ejemplos. El primero corresponde al momento en que Eduard está ocupado en colocar los fuegos artificiales con los que se celebrará el cumpleaños de Ottilie. Proyecta adecentar el espacio bajo los plátanos, enfrente del cual, al otro lado del agua y bajo unos robles, se encenderán los fuegos:

Unter einem andern Vorwand ließ daher Eduard den Raum unter den Platanen von Gesträuch, Gras und Moos säubern, und nun erschien erst die Herrlichkeit des Baumwuchses sowohl an Höhe als Breite auf dem gereinigten Boden. Eduard empfand darüber die größte Freude. 'Es war ungefähr um diese Jahreszeit, als ich sie pflanzte. Wie lange mag es her sein?' sagte er zu sich selbst. Sobald er nach Hause kam, schlug er in alten Tagebücher nach, die sein Vater, besonders auf dem Lande, sehr ordentlich geführt hatte. Zwar diese Pflanzung konnte nicht darin erwähnt sein, aber eine andre häuslich wichtige Begebenheit an demselben Tage, deren sich Eduard noch wohl erinnerte, mußte notwendig darin angemerkt stehen. Er durchblättert einige Bände, der Umstand findet sich. Aber wie erstaunt, wie erfreut sich Eduard, als er das wunderbarste Zusammentreffen bemerkt! Der Tag, das Jahr jener Baumpflanzung ist zugleich der Tag, das Jahr von Ottiliens Geburt (Goethe 2009: 99).

Los trabajos en el lugar tienen como resultado una nueva apreciación del tamaño y de la grandeza de los plátanos. Este nuevo conocimiento despierta la sed de más, de saber cuándo fueron plantados. Un deseo que le lleva a consultar los diarios de su padre, que funcionan como archivo de las actuaciones en los terrenos de la propiedad. Una crónica en forma de diario: conocimiento coleccionado, 
sistematizado, gracias al cual Eduard puede deducir que los plátanos fueron plantados en el mismo día en el que nació Otilie.

Vemos, pues, de qué forma sutil teje Goethe conocimiento y naturaleza en la materialidad del jardín, incluso en los momentos relacionados de forma menos evidente con el coleccionismo -como lo serían la colección de grabados del arquitecto o las historias del viajero inglés-. Una forma de enhebrar circunstancias que lleva al lector a ir conociendo nuevas informaciones, realidades o estados de los personajes de la novela. Por todo ello el jardín -y la novela en sí- puede ser considerado una heterotopía, en el sentido apuntado, a modo de conclusión, en el epígrafe siguiente.

\section{Conclusión: heterotopía y estética del jardín}

El 14 de marzo de 1967 en el Cercle d'études architecturales, Michel Foucault comenzó su conferencia Des espaces autres caracterizando al siglo XIX como el siglo de la historia, esto es, del tiempo, frente al siglo XX, en el que el centro de la reflexión sería el espacio (Foucault 1994: 752). Es una caracterización que no podemos dejar de rechazar a la vista de lo analizado hasta ahora. Pero, más allá de esta diferenciación un tanto falaz, sí coincidimos con la afirmación con la que a continuación Foucault empieza a analizar la materia de la que tratará su conferencia: 'l'espace lui-même, dans l'expérience occidentale, a une histoire, et il n'est pas possible de méconnaître cet entrecroisement fatal du temps avec l'espace" (Foucault 1994: 753). No entramos en la caracterización de "fatal" de este entrecruzamiento entre tiempo y espacio, sino que nos limitamos a señalar -en la línea de lo dicho hasta ahora- en la observación de que el espacio tiene historia $y$ diégesis. Y viceversa, añadiríamos: la historia, la diégesis, tiene su espacio. En nuestro caso, el jardín.

El jardín es precisamente uno de los casos que Foucault nombra cuando ejemplifica el concepto de heterotopía, que lanza en esa conferencia, definiéndola de la siguiente manera (Foucault 1994: 755-756):

Il y a également, et ceci probablement dans toute culture, dans toute civilisation, des lieux réels, des lieux effectifs, des lieux qui sont dessinés dans l'institution même de la société, et qui sont des sortes de contre-emplacements, sortes d'utopies effectivement réalisées dans lesquelles les emplacements réels [...] que l'on peut trouver à l'intérieur de la culture sont à la fois représentés, contestés et inversés, des sortes de lieux qui sont hors de tous les lieux, bien que pourtant ils soient effectivement localisables. Ces lieux, parce qu'ils reflètent et dont ils parlent, je les appellerai, par opposition aux utopies, les hétérotopies; et je crois que entre les utopies et ces emplacements absoluement autres, ces hétérotopies, il y aurait sans doute une sorte d'expérience mixte, mitoyenne, qui serait le miroir. Le miroir, après tout, c'est une utopie, puisque c'est un lieu sans lieu. [...] Mais c'est également une hétérotopie, [...]. [...] le miroir fonctionne comme une hétérotopie en ce sens qu'il rend cette place que j'occupe au moment où je me régarde dans la glace, à la fois absolument 
réelle, en liaison avec tout l'espace qui l'entoure, et absoluement iréelle, puisqu'elle est obligée, pour être perçue, de passer par ce point virtuel qui est là bas.

El jardín participa de las dos propiedades que le asigna aquí Foucault al espejo. Es más, podemos afirmar que el jardín en Die Wahlverwandtschaften funciona como un espejo temporalizado: en tanto que creación, construcción, el jardín es la proyección de un deseo (Vogl 2008: 299) y en este sentido, utopía; en tanto que realidad percibida por los protagonistas de la novela, el jardín retrata sus individualidades por medio de los deseos de reforma que despierta en ellos la contemplación de lo realizado en el jardín; esta virtualidad del jardín pone de manifiesto su carácter tan especular como heterotópico.

Pero no por tener carácter especular, el jardín en Die Wahlverwandtschaften deja de ser lo que es: un jardín. Como tal ejemplifica el tercero de los principios de descripción de una heterotopía que Foucault establece: "L’hétérotopie a le pouvoir de juxtaposer en un seul lieu réel plusieurs espaces, plusieurs emplacements, qui sont en eux-mêmes incompatibles" (Foucault 1994: 758). Es a todas luces clara la relación que tiene esta descripción de la heterotopía con la realidad del jardín en Die Wahlverwandtschaften: el jardín en torno al castillo del barón Eduard es un lugar real -los dominios del noble- en el que se distinguen desde el principio de la novela tres lugares en principio incompatibles, pero que son partes de la misma totalidad: el huerto, el jardín inglés y el cementerio. Una observación que se ve confirmada por ser precisamente uno de los casos con los que ejemplifica este principio descriptivo Foucault (Foucault 1994: 758-759):

[...] l'exemple plus ancien de ces hétérotopies, en forme d'emplacements contradictoires [...] c'est peut-être le jardin. Il ne faut pas oublier que le jardin, étonnante création maintenant millénaire, avait en Orient des significations très profondes et comme superposées. Le jardin traditionnel des Perses était un espace sacré qui devait réunir à l'intérieur de son rectangle quatre parties représentant les quatres parties du monde, avec un espace plus sacré encore que les autres qui était comme l'omblic [...]; et toute la végétation du jardin devait se répartir dans cet espace, dans cette sorte de microcosme. Quant aux tapis, ils étaient, à l'origine, des reproductions de jardins. Le jardin, c'est un tapis où le monde tout entier vient accomplier sa perfection symbolique, et le tapis, c'est une sorte de jardin mobile à travers l'espace. Le jardin, c'est la plus petite parcelle du monde et puis c'est la totalité du monde. Le jardin, c'est, depuis le fond de l'Antiquité, une sorte d'hétérotopie heureuse et universalisante (de là nos jardins zoologiques).

Vemos, por tanto, que la caracterización que Foucault hace del jardín como heterotopía confirma y en cierto modo resume lo que hemos venido observando y sosteniendo: al jardín, entendido como heterotopía, le acompaña una pretensión universalizante de reproducir el mundo. En el caso de Die Wahlverwandtschaften esta inclinación no busca recoger las cuatro partes del mundo, sino dos mundos: el huerto, la industria heredada por Eduard, y el paisaje ideado por Charlotte. La relación que cada uno de estos personajes -a los que añadiríamos al mayor y a Ottilie- con cada una de las partes del jardín es simbólica. En los terrenos del barón 
Eduardo lindan dos tipos de estéticas del jardín, dos modos de percepción del mismo: el industrioso del huerto y el contemplativo del jardín inglés. De forma análoga al modo en que, al final de la novela, descansan en paz, tumba con tumba, los cuerpos yertos de dos individualidades patéticas: la del activo Eduard y la de la contemplativa Ottilie.

\section{Referencias bibliográficas}

Bolz, N., «Die Wahlverwandtschaften», en: Goethe-Handbuch, Bd. 3: Prosaschriften. Weimar: Metzler 1997.

CORTÉS GABAUDAN, H., «La arquitectura de jardines como correlato a las relaciones sentimentales en Die Wahlverwandtschaften de Goethe», en: SiguAN, M./JANÉ, J. (eds.), Ihr mögt micht benutzen. Barcelona: Sociedad Goethe de España 2004, 37-52.

DeleUZE, G., Michel Foucault. Paris: Minuit 1986.

Foucault, M., «Des espaces autres», en: Foucault, M. (eds.), Dits et écrits. Vol. 4 (1980-1988). Paris: Gallimard 1994, 752-762.

FoucAult, M., Les mots et les choses: une archéologie des sciences humaines. Paris: Gallimard 1966.

Foucault, M., Naissance de la biopolitique: cours au Collège de France (1978-1979). Paris: Gallimard-Seuil 2004.

GerndT, O., Sozialer Strukturwandel in Goethes Literatur der 1790er Jahre: eine literaturso-ziologische Untersuchung. Konstanz: Univ. Diss. 1991.

GoETHE, J. W., Sämtliche Werke. Münchner Ausgabe (MA) in 14 Bände. München: dtv 1977.

GOETHE, J. W., Die Wahlverwandtschaften. München: dtv $2009^{14}$

GROß, S., Die Weimarer Klassik und die Gartenkunst. Über den Gattungsdiskurs und die "Bildenden Künste" in den theoretischen Schriften von Goethe, Schiller und Krause. Frankfurt a. Main u.a.: Peter Lang 2009.

HABERL, H., «'An der Natur versuchen': Landschaft, Garten und die Grenzen der heiteren Vernunftfreiheit in Die Wahlverwandtschaften und Bouvard et Pécuchet», en: KRINGS, M. (Hg.), Deutsch-französische Literaturbeziehungen: Stationen und Aspekte dichterischer Nachbarschaft vom Mittelalter bis zur Gegenwart. Würzburg: Königshausen \& Neumann 2007, 171-185.

LEPENIES, W., Das Ende der Naturgeschichte. Wandel kultureller Selbstverständlichkeiten in den Wissenschaften des 18. und 19. Jahrhunderts. München: Hanser 1976.

Mallarme, S., La musique et les lettres. Paris: Perrin et Cie. 1895.

MATUSSEK, P., «Formen der Verzeitlichung. Der Wandel des Faustschen Naturbildes und seine historische Hintergründe», en: MATUSSEK, P. (Hg.), Goethe und die Verzeitlichung der Natur. München: Beck 1998, 202-232.

Niedermeier, M., Das Ende der Idylle. Symbolik, Zeitbezug, Gartenrevolution in Goethes Roman "Die Wahlverwandtschaften". Frankfurt a. Main u.a.: Peter Lang 1992.

POLIANSKI, I., Die Kunst, die Natur vorzustellen. Die Ästhetisierung der Pflanzenkunde um 1800 und Goethes Gründung des botanischen Gartens zu Jena im Spannunfeld kunsttheore- tischer und botanischer Diskussionen der Zeit. Köln: König 2004.

PONZI, M., «Arte e natura: il cambio di paradigma nella poetica di Goethe», Links 7 (2007), 13-36

Sampaolo, G., Prosepinens Park. Goethes "Wahlverwandtschaften" als Selbstkritik der Moderne. Weimar: Metzler 2003. 
SCHNEIDER, H., «Dichter, Herrscher, Natur: Die Entstehung des Ilmparks und das Bild des Parks in Goethes Dichtung», Goethe Yearbook 12 (2004), 93-109.

TABARASI, A.-S., Der Landschaftsgarten als Lebensmodell: zur Symbolik der “Gartenrevolution” in Europa. Würzburg: Königshausen \& Neumann 2007.

TRAEGER, J., «Goethes Kunstwelten: vom Landschaftsgarten zum lebenden Bild», en: VAlEnTIN, J.-M., Johann Wolfgang Goethe zum 250. Geburtstag: Vorträge im Frankfurter Römer. Paris: Didier Erudition 1999.

VoGL, J., Kalkül und Leidenschaft. Poetik des ökonomischen Menschen. Zürich-Berlin: Diaphanes 2008.

ZABKA, T., "Goethes ästhetische Integration von Natur- und Gesellschaftsidee», en: MatusseK, P. (Hg.), Goethe und die Verzeitlichung der Natur. München: Beck 1998, 157-177. 